\title{
P01-2-33 Poster session
}

\section{Cadmium induces dose-dependent cardiovascular fates with differential underlying hemodynamic, neural and cellular mechanisms}

\author{
Ching-Yi Tsai, Samuel H. H. Chan \\ Institute for Translational Research in Biomedicine, Chang Gung Memorial Hospital, Taiwan
}

Cadmium is a heavy metal and an environmental toxicant. Acute exposure to cadmium induces cardiovascular toxicity and mortality. This study aims at determining whether lethal or sub-lethal dose of cadmium induces cardiovascular toxicity on the heart, blood vessels or neural substrates of the baroreflex via different mechanisms.

In male Sprague-Dawley rats maintained under propofol anesthesia, intravenous administration of cadmium (4 mg/kg, i.v.) resulted in a gradual decrease in mean arterial pressure (MAP), heart rate (HR) and power density of the lowfrequency (BLF) component, an index for baroreflex-mediated sympathetic vasomotor tone, which only became significant $180 \mathrm{~min}$ after injection. On the other hand, cadmium $(8 \mathrm{mg} / \mathrm{kg})$ elicited a significantly and abruptly diminished MAP, HR and BLF power, leading to death within 20 min. Closer scrutiny of the first 5 min (Fig. 1) after administration of cadmium $(4 \mathrm{mg} / \mathrm{kg}$, i.v. ) showed a transient decrease in MAP that was mirrored by an increase in carotid blood flow and BLF power, along minor changes in HR and renal or femoral blood flow. On injection of cadmium $(8 \mathrm{mg} / \mathrm{kg}$, i.v. $)$, the transient decrease in MAP followed a continuous trend towards zero level, as were the renal and femoral blood flow. The transient increase in carotid blood flow soon gave way to a decrease, alongside irregular changes in BLF power. Echocardiography and pressure-volume loop analysis further showed dose-dependent decrease in cardiac contractility. Tractographic analysis with DTI of the brain stem demonstrated that the connectivity between nucleus tractus solitarii (NTS) and rostral ventrolateral medulla (RVLM) was disrupted or severed coincidental with the decrease or loss of baroreflex-mediated sympathetic vasomotor tone that accompanied the sub-lethal or lethal dose of cadmium. These neuroimaging data were corroborated by the exhibition in the NTS or RVLM of caspase-related apoptosis induced via mitochondrial dysfunction by the low dose, or necrosis induced via bioenergetics failure because of hypoxia by the high dose of cadmium.

We conclude that cadmium elicits drastically different cardiovascular fates that are dose-dependent, which entail differential underlying hemodynamic (cardiac contractility, cerebral autoregulation, regional blood flow), neural (baroreflex) and cellular (mitochondrial dysfunction, apoptosis, bioenergetics failure, necrosis) mechanisms. 